\title{
ALCOHOL USE BY YOUTH IN THE CZECH REPUBLIC AND FINLAND: AN EMPIRICAL TEST OF SKOG'S THEORY OF THE DISTRIBUTION OF ALCOHOL CONSUMPTION ${ }^{1}$
}

\author{
IVETA ČERMÁKOVÁ \\ Department of Sociology, Faculty of Arts, Charles University \\ E-mail: cermakova.iw@gmail.com \\ ZUZANA PODANÁ \\ Department of Sociology, Faculty of Arts, Charles University \\ E-mail: zuzana.podana@ff.cuni.cz
}

\begin{abstract}
This article focuses on alcohol consumption among Czech and Finnish youth. First, it describes the situation regarding alcohol consumption in both countries, including trends in consumption in recent years, and, second, it presents and tests the theory of the distribution of alcohol consumption by a Norwegian sociologist Ole-Jørgen Skog, which has had an influence on many alcohol policies. Skog's theory describes the relationship between mean alcohol consumption in a population and the consumption at all levels of consumption (from light to heavy drinkers) and suggests that they are strongly interconnected and are of log-linear nature. Data used in this study come from two large-scale international surveys the "European School Survey Project on Alcohol and Other Drugs" and the "International Self-Report Delinquency" study and cover the period from 2003 to 2013. Results show that alcohol consumption among Finnish juveniles is considerably lower compared to their Czech peers and, moreover, it seems to gradually decrease in the given time period, again, unlike Czech youth. Results concerning the test of Skog's theory are largely consistent with his propositions. The only difference is revealed, specifically it is the group of heavy drinkers - not light drinkers as in Skog's study - which is most affected by changes in mean alcohol consumption in the population.
\end{abstract}

Key words: youth; alcohol; Skog's theory; Czech Republic; Finland

\section{Introduction}

Consumption of alcohol by children and adolescents is a persistent problem which is often targeted by alcohol policies and countries set different preventive measures to deal with this issue. The tradition of production and consumption of alcohol is very strong in the Czech Republic and the cultivation of raw materials and the production of alcoholic beverages is an important industrial sector as well. Together with low legal regulations regarding the sale and consumption of alcohol, and general tolerance to consumption of alcoholic beverages, people in Czech society are socialized into an environment where alcohol consumption is a commonplace. No wonder then that

1 The study is based on results of Iveta Čermákovás (2016) master thesis. The preparation of this journal article was supported by the Charles University funding scheme Progres Q15.

Commons Attribution License (http://creativecommons.org/licenses/by/4.0). 
the Czech Republic ranks among the countries with the highest prevalence of alcohol consumption by adults and even youth (World Health Organization, 2014; Hibell et al., 2012; Kraus et al., 2016).

The main objectives of this article are, first, to describe the situation with respect to alcohol consumption by youth in the Czech Republic and Finland, including the development in recent years. Second, we intend to look at the issue of alcohol consumption among youth through the theory of the distribution of alcohol consumption by Norwegian sociologist Ole-Jørgen Skog (1985), who claims that alcohol consumption in a society has a very strong collective character and the mean consumption of alcohol in a society affects consumers at all levels of consumption (from light to heavy drinkers). To empirically test this theory, we use data from two international surveys which focused at least partly - on alcohol consumption of youth, namely the "European School Survey Project on Alcohol and Other Drugs" (ESPAD) and the "International Self-Report Delinquency" study (ISRD).

The analysis presented in this article is conducted on data from the Czech Republic and Finland. The reason for the inclusion of a second country as a comparison is mainly methodological. For a better verification of Skog's theory, it is convenient to test the model on data from countries, which have different trends in alcohol consumption as well as different prevalence rates of consumption. (e.g., Hibell et al., 2012).

\section{Skog's theory of the distribution of alcohol consumption}

According to our knowledge, Skog's (1985) theory has rarely been addressed by Czech researchers (e.g. Kubička et al., 1998), although it is quite significant in the field of alcohol use and it has influenced many alcohol policies. Its fundamental pillar is that the average alcohol consumption in the society has a significant effect on the individual consumption. Skog formulated two hypotheses about human drinking behavior in the framework of his theory:

\section{Hypothesis of the multiplicativity of effects}

Individual behavior in the area of alcohol consumption is influenced on the one hand by biological and psychological factors and on the other hand by environmental factors. In short, individual alcohol consumption behavior is influenced by a large number of more or less dependent factors, which combine and recombine themselves to create such behavior. The factors affecting the individual drinking behavior mutually interact and tend to have a multiplicative effect. The hypothesis of the multiplicativity of effects was tested by Skog (1985) on panel data and his conclusion was that the relationship between the individual level of consumption in two following years, was indeed log-linear (i.e. multiplicative).

\section{Hypothesis of the mechanism of social interaction}

Drinking behavior and habits are strongly influenced by friends, more generally, by personal social networks, where family or friends influence the drinking behavior directly or indirectly.

These two above described hypotheses lead to these two consequences: 


\section{1a. The consequence of the multiplicativity of effects - skewness of the distribution}

The multiplicativity of effects results in a skewness of the distribution of alcohol consumption in which the arithmetic mean is usually considerably higher than the median. Skog (1985) analyzed data from 9 different countries, which consisted of 21 populations. His conclusion was that the average consumption was typically double compared to the median in all populations. Nevertheless, the skewness is generally less pronounced in populations with a high average consumption of alcohol. Skog also challenged the idea that the end of the distribution of alcohol consumption comprises only a small group of extreme cases, i.e. alcoholics. It is clear that alcoholics cannot be the reason for the skewness of the distribution, and therefore the distribution of "normal drinkers" must be skewed as well.

2a. The consequence of the mechanism of social interaction - collectivity of drinking cultures

Since individual drinking behavior is influenced by social networks, both directly and indirectly, changes in drinking behavior are necessarily a group phenomenon. The whole population can be considered a huge social network, since it is a system of actors, who are bound by different social relations that coordinate their behavior. Each actor is influenced by a small group of his acquaintances, but he is also influenced indirectly by a large group of other people. Thus, each individual is directly or indirectly affected by every member of his own culture. The strength of ties in the social network affects the speed and efficiency of the transmission of impulses, leading to synchronized changes of social groups throughout the society. On this basis, Skog (1985) builds the foundation of his theory and proposes that such a synchronization also appears in social networks of alcohol drinkers. Consequently, each individual adapts his individual alcohol consumption behavior to the mean level of consumption in his culture.

On the basis of his empirical data analysis, Skog (1985) concluded that the collective drinking culture exists. Moreover, if there are changes in consumption, we can expect that they also include changes in consumption patterns and habits among consumers at all levels of consumption. Therefore, drinking culture cannot be seen as an aggregate of independent individuals, but rather as a highly-organized system of interconnected actors. It follows that statistical variable "mean alcohol consumption" has a sociocultural context.

Figure 1 summarizes results of the Skog's (1985) analysis. The graph shows the relationship between the mean consumption in the population and consumption levels of light, moderate, medium, nearly heavy and heavy drinkers (these groups defined on the basis of the percentile values). An increase in the mean alcohol consumption in a population is associated with an increase in consumption in all groups of drinkers. In this way, "population moves in concert upwards along the consumption scale, and drinking in effect seems to have a very strong collective component." (ibid: 90). The chart suggests that relationships between variables can be represented by a log-linear model, which supports the hypothesis of the multiplicativity of effects.

Skog (1985) did not find the strength of the multiplicativity of effects in all groups of consumers the same. His results showed that the highest increase in consumption is among light and moderate drinkers. This fact is reflected in the slope of the regression curve - the steeper, the greater the increase in consumption in the group of consumers. 


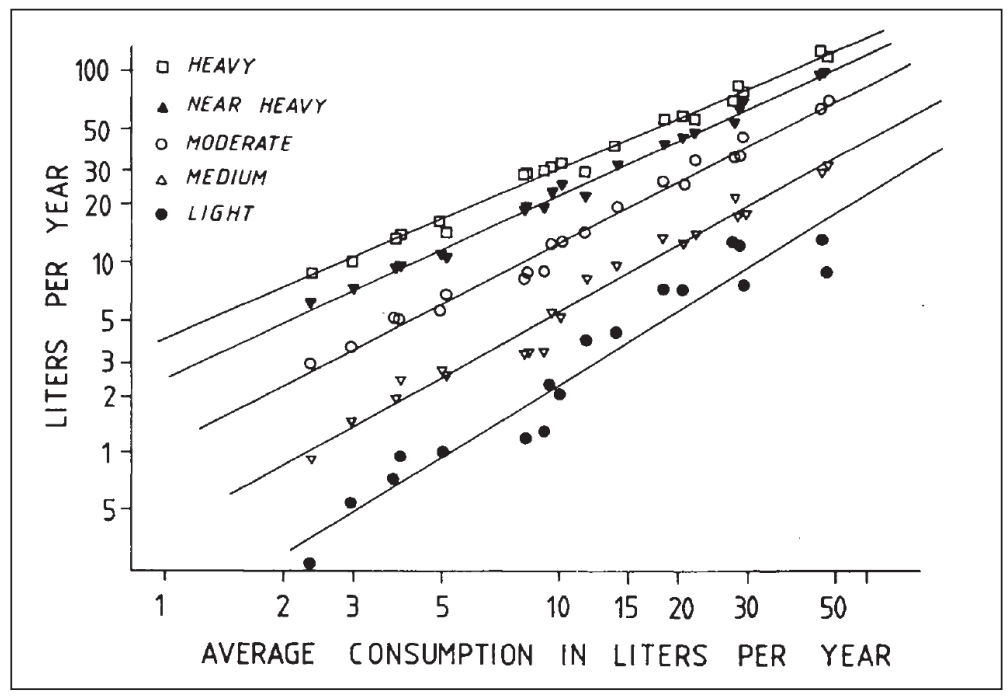

Figure 1: Skog's results on the relationship between average consumption and the consumption level of 21 drinking groups (defined by percentiles)

Note: $\log$ values reported

Source: Skog, 1985: 90

Furthermore Skog (1985) describes the relationship between mean alcohol consumption and heavy drinking. Based on the fundamental premise that the entire population moves upward the consumption scale if the mean consumption increases, the number of people over a certain consumption limit should increase as well. Therefore, there should be a relationship between the mean consumption in the population and the prevalence of heavy drinkers. This proposition has an implication for alcohol policies as well and suggests that an effective effort to reduce the total consumption of alcohol will also lead to a reduction in heavy drinking and alcohol related harm.

Despite its publication in 1980s, Skog's (1985) theory has been of great interest in recent years. Several studies tried to empirically re-test this theory (e.g. Gmel \& Rehm, 2000; Rossow, Mäkelä, \& Kerr, 2014; Brunborg, Bye, \& Rossow, 2014) and yielded mixed results - it was supported by some of these studies but not by others. An interesting debate about the validity of Skog's theory has been lately produced by researchers mainly from Scandinavian countries (e.g. Bjarnason, 2006; Livingston, 2008; Meier, 2010). There is a development in alcohol consumption by youth in several countries which contradicts Skog's theory - while youth alcohol consumption declines, the prevalence of heavy drinkers of alcohol increases. Thus, it is rather a polarization between light and heavy drinkers than a collective change (e.g. Bjarnason, 2006). Furthermore, two recent studies among Swedish youth brought different results in testing Skog's theory. Results by Hallgren, Leifman and Andreasson (2012) refuted it, while Norström and Svensson (2014) reported findings supportive of Skog's theory. 


\section{Present study}

The first objective of this study is to describe the situation in juvenile alcohol consumption in the Czech Republic and Finland and examine its development in recent years. Data used in this study come from two large-scale international projects, namely ESPAD and ISRD, which is advantageous due to the fact that they comprise different age groups and use somewhat different indicators. Hence, the resulting picture is more comprehensive.

Second, Skog's (1985) theory of the distribution of alcohol consumption is empirically tested on the data from ESPAD. Specifically, we aim at an evaluation of following propositions derived from this theory:

1. The distribution of alcohol consumption is asymmetric in both countries.

2. A change in mean alcohol consumption is associated with a change in the proportion of strong drinkers.

3. A change in mean alcohol consumption is associated with a change in all groups of consumers.

Third, the data from the ISRD survey is used for a partial test of Skog's (1985) theory (Proposition 3) employing a different indicator of alcohol consumption. As ISRD does not include information about the exact amount of consumed alcohol, the number of drinking occasions in the last month is utilized instead. This approach enables us to judge if this Skog's assumption is valid even if the frequency of drinking is analyzed.

\section{Methods}

\section{Data}

Data from two international projects focusing on youth are utilized in this article. The first one is the ESPAD survey which is a continual research project carried out in various European countries and its main goal is to collect data on substance use among European teenagers aged 15-16 years. Large school-based samples are drawn every four years and, among others, allow to study trends in alcohol consumption among juveniles (see e.g. Csémy, Lejčková, Sadílek, \& Sovinová, 2006; Hibell et al., 2012). Datasets used in the subsequent analyses come from the Czech Republic and Finland from years 2003, 2007, and 2011.

The second datasets come from the ISRD project which is an international research of delinquency and victimization of youth which inquire about alcohol consumption as well. This school-based survey focuses on children from seventh, eighth, and ninth grades of school, i.e. aged approximately twelve to fifteen years (see e.g., ISRD3 Working Group, 2013; Moravcová, Podaná, \& Buriánek, 2015; Junger-Tas et al., 2010). Datasets analyzed in this article come from the Czech Republic and Finland from two sweeps of the project which were held in 2006 (and partly 2007) and 2013. Due to differences in sampling procedures between the countries, the samples used in analysis include only juveniles from large cities. In addition, the samples were restricted to children aged 13-15 years. 


\section{Measures of alcohol consumption}

For descriptive purposes, simple prevalence indicators of alcohol consumption during life-time, the last year, and the last month and the prevalence of binge drinking ${ }^{2}$ during the last month are used. In addition, for the purpose of a test of Skog's (1985) theory, two "alcohol indices" are computed, one for the ESPAD data and the other one for the ISRD data.

First, ESPAD alcohol index is described. We utilize the question: "On how many occasions (if any) have you had any alcoholic beverage to drink... during the last 30 days?" Response options reflected the number of opportunities for alcohol consumption and were the following: "never" (code 0), "1-2" (code 1.5), "3-5" (code 4), "6-9" (code 7.5), "10-19” (code 14.5), “20-39” (code 30), “40 or more times” (code 30). This question was chosen as an estimate of the frequency of alcohol consumption in the last 30 days. Response options were recoded for the analysis to the number of days in the month.

To express the amount of alcohol consumed during the last occasion, we included the following block of questions from the questionnaire: "If you drank beer/cider/alcopops/ wine/spirits that last day you drank any alcohol, how much did you drink?" Response options for beer, cider and alcopops in the questionnaire ESPAD 2007 and 2011 were the following: "I never drink beer/cider/alcopops" (code 0), "I did not drink beer/cider/ alcopops on the last day that I drank alcohol" (code 0), "<50 cl" (code 25), "50-100 cl" (code 75), "101-200 cl” (code 150), “>200 cl” (code 200). Answer options on wine and spirits were different in questionnaires 2007 and 2011 from the questionnaire of 2003 which were stated: "I never drink wine" (code 0), "I did not drink wine on the last day that I drank alcohol” (code 0), “<15 cl” (code 7.5), "15-30 cl” code (22.5), "37 cl” (code 37 ), ">75 cl" (code 75). In the questionnaires of the years 2007 and 2011, the first two options were the same as in 2003 (both coded as 0 ), else: "<20 cl" (code 10), "20-40 cl" (code 30), "41-74 cl” (code 58), ">74" (code 74). In the case of spirits, the first two options were the same in all questionnaires as in the previous types of alcohol: "I never drink spirits" (code 0), "I did not drink spirits on the last day that I drank alcohol "(code 0 ), the other options in 2003 were: " $<5 \mathrm{cl}$ " (code 2.5), " $5-10 \mathrm{cl}$ " (code 7.5), "11-25 cl" (code 18), ">30 cl" (code 30). In the questionnaires 2007 and 2011, as follows: " $<7 \mathrm{cl}$ " (code 4) $)^{3}$, "8-15 cl” (code 11.5), "16-24 cl” (code 20), ">25 cl” (code 25) ${ }^{4}$. We include only respondents who answered at least 3 questions (out of a total of 5 types of alcohol) into the following analysis. ${ }^{6}$

The amount of alcohol consumed during the last month (alcohol index) was constructed as the sum of the amount of alcohol consumed during the last occasion for each

2 Binge drinking (or heavy episodic drinking) was defined as having at least 5 drinks on one occasion (in ESPAD survey).

3 In questionnaire ESPAD 2011 was this option " $<8 \mathrm{cl}$ " (code 4$)$.

4 In questionnaire ESPAD 2011 was this option " $>24 \mathrm{cl}$ " (code 24).

5 The codes listed in brackets indicate the centers of the intervals of the drunk amount of given type of alcohol. If the last option was chosen, the code is the given value.

6 It should be also noted that a question on the consumption of alcopops was not included in the Czech or the Finnish version of the questionnaire from 2003. The questions on the consumption of cider and alcopops were marked as optional in 2007 and 2011. The question on cider was not included in any of Czech questionnaires as cider was rarely consumed in the Czech Republic in this time period. 
respondent multiplied by the number of occasions the respondent drank during the last month. The amount of alcohol consumed for different types of alcohol was converted to centiliters of pure alcohol by the following logic: ${ }^{7}$

- amount of beer/alcopops/cider during the last occasion * ${ }^{*} .5$

- amount of wine during the last occasion * .14

- amount of spirits during the last occasion * .30

Second, a different alcohol index was computed for the ISRD data and it was based on the following questions:

- ISRD-2: "Did you drink (beer/wine/spirits) during the last 4 weeks? If yes how many times?"

- ISRD-3: "Think back over the LAST 30 DAYS. On how many occasions (if any) have you had any of the following to drink?" (beer/wine/spirits).

Another complication was the question about the number of opportunities of beer and wine consumption. Whereas ISRD-2 inquired about both types of alcohol together in a single question, there were two separate questions in ISRD-3. Final alcohol index was compute for each respondent as a sum of the highest number of opportunities from all these types of alcoholic beverages (beer/wine/spirits) and the number of the other opportunities for the other alcoholic beverages divided by two.

\section{Results}

First, we concentrate on a description of the situation in alcohol consumption among juveniles in the Czech Republic and Finland (Table 1-2). Results from both ESPAD and ISRD show a striking difference in the levels of alcohol consumption between Czech and Finnish youth - Czech juveniles are considerably more likely to drink alcohol than their Finnish peers, no matter which indicator is used for the comparison. The data from three sweeps of ESPAD (2003-2011) for children 15-16 years old show a stable trend or even an increase in consumption (last month prevalence and binging) among Czech youth (see Table 1). On the contrary, the trend for Finnish juveniles gradually decreases in case of both life-time and last year prevalence and a considerable decrease in the last month prevalence and binge drinking occurred between 2003 and 2007. ${ }^{8}$ Furthermore, if the ISRD data from 2006 and 2013 are considered (see Table 2) which comprise age categories 13-15, the decline in life-time prevalence of Finnish youth is even more pronounced and the increase in the last month prevalence of Czech youth is also present, even surprisingly large.

7 Volume of alcohol of drink types was chosen from this source: http://www.oecd.org/health/health -data.htm; https://en.wikipedia.org/wiki/Alcoholic_beverage

8 The data from a subsequent sweep of ESPAD from 2015 show a continuous large decrease in alcohol consumption among Finnish youth but, surprisingly, a moderate decrease is found also among Czech juveniles (Chomynová, Csémy, \& Mravč́́k, 2016; Kraus et al., 2016). 
Table 1: Prevalence of alcohol consumption among Czech and Finnish adolescents 15-16 years-old (\%)

\begin{tabular}{|l|c|c|c|c|c|c|}
\hline & \multicolumn{3}{|c|}{ Czech Republic } & \multicolumn{3}{c|}{ Finland } \\
\hline & $\mathbf{2 0 0 3}$ & $\mathbf{2 0 0 7}$ & $\mathbf{2 0 1 1}$ & $\mathbf{2 0 0 3}$ & $\mathbf{2 0 0 7}$ & $\mathbf{2 0 1 1}$ \\
\hline Life-time prevalence & $\mathbf{9 8 . 4}$ & $\mathbf{9 7 . 4}$ & 97.9 & $\mathbf{8 7 . 9}$ & $\mathbf{8 5 . 4}$ & $\mathbf{8 3 . 6}$ \\
\hline Last year prevalence & $\mathbf{9 4 . 9}$ & $\mathbf{9 3 . 2}$ & 93.1 & $\mathbf{7 9 . 9}$ & $\mathbf{7 6 . 5}$ & 74.9 \\
\hline Last month prevalence & 77.0 & $\mathbf{7 5 . 6}$ & $\mathbf{7 9 . 0}$ & $\mathbf{5 4 . 4}$ & $\mathbf{4 7 . 9}$ & 47.8 \\
\hline Binging - last month prev. & $\mathbf{4 7 . 3}$ & $\mathbf{5 1 . 5}$ & 53.7 & $\mathbf{4 0 . 2}$ & $\mathbf{3 4 . 0}$ & 34.7 \\
\hline N & 3172 & 3901 & 3913 & 3222 & 4988 & 3744 \\
\hline
\end{tabular}

Note: significant differences ( $\mathrm{p}$ <.05) between 2003 and 2007 and between 2007 and 2011 within each country marked in bold; all differences are significant between countries within all 3 years Source: ESPAD

Table 2: Prevalence of alcohol consumption among Czech and Finnish adolescents 12-15 years-old (\%)

\begin{tabular}{|l|c|c|c|c|}
\hline & \multicolumn{2}{|c|}{ Czech Republic } & \multicolumn{2}{c|}{ Finland } \\
\hline & $\mathbf{2 0 0 6}$ & $\mathbf{2 0 1 3}$ & $\mathbf{2 0 0 6}$ & $\mathbf{2 0 1 3}$ \\
\hline Life-time prevalence & 85.2 & 87.5 & $\mathbf{6 9 . 3}$ & $\mathbf{5 4 . 6}$ \\
\hline Last month prevalence & $\mathbf{4 1 . 9}$ & $\mathbf{7 1 . 3}$ & 30.4 & 31.8 \\
\hline $\mathrm{N}$ & 1035 & 1468 & 1212 & 2058 \\
\hline
\end{tabular}

Note: significant differences ( $\mathrm{p}$ <.05) between 2006 and 2013 in CZ (FI) marked in bold; all differences are significant between countries within both years

Source: ISRD

Next, we approach to the test of Skog's (1985) theory. For this purpose, we use the ESPAD data and include only those adolescents who drank alcohol in the last 30 days into the analysis (see, Brunborg, Bye, \& Rossow, 2014). On the basis of the values of alcohol index, we can confirm Skog's (1985) assumption that alcohol consumption is distributed asymmetrically in both populations and it is strongly skewed to the right (see Figure 2). Furthermore, the distribution of alcohol consumption is different in both compared countries, specifically, the distribution of alcohol consumption in Finland is more skewed.

In the next step, we categorized juveniles into 5 groups based on their consumption levels in comparison to their peers (numbers indicate the percentile values of each group): 25 - light drinkers; 50 - medium drinkers; 75 - moderate drinkers; 90 - near heavy drinkers; 95 - heavy drinkers. A juvenile who drinks the amount of the $25^{\text {th }}$ percentile value (i.e. $25 \%$ of drinkers consume less alcohol than he/she) is a typical representative of the group, which we, according to Skog, label light drinkers (similarly in all other groups). Then, the original data from the years 2003, 2007, and 2011 were aggregated into groups by the year of the survey, country, gender, and the percentile value. The resulting data set contains following indicators for each group of drinkers: the value of the alcohol index, the value of mean alcohol consumption and also log values of these variables.

If the results are inspected (Table 3), it is clear that the mean alcohol consumption among Finnish youth (who consume alcohol) slightly increases in time. The increase in 


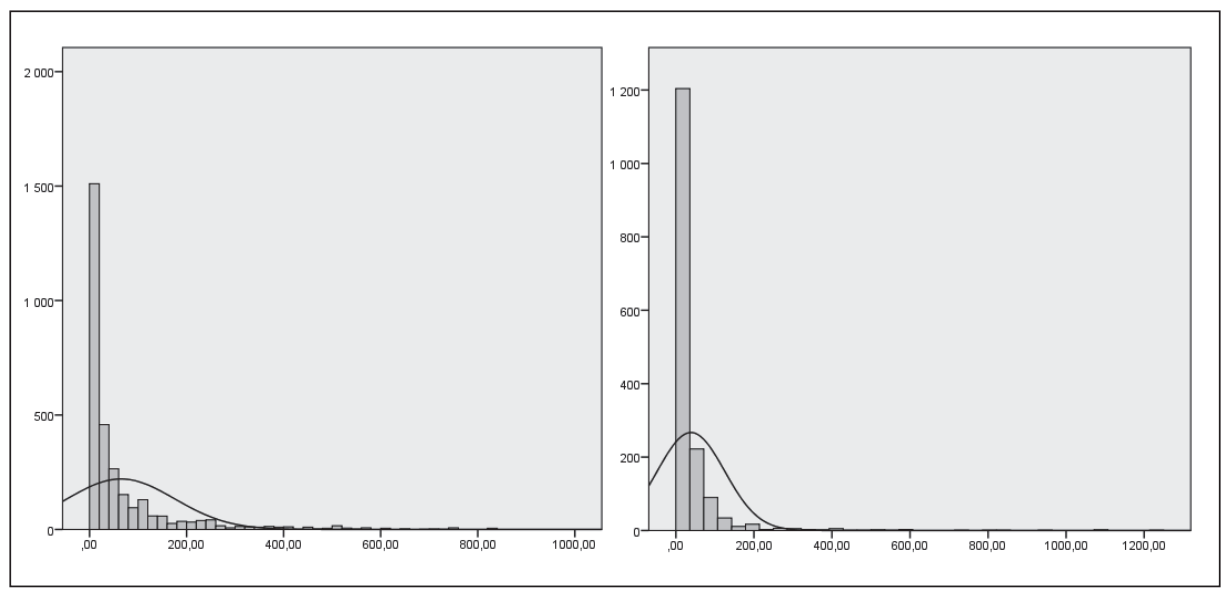

Figure 2: Distribution of alcohol index in the Czech Republic and Finland

Note: $\mathrm{CZ}$ on the left $-\mathrm{N}=3066$, skewness $=3.7$; S.E. $=.03$; FI on the right $-\mathrm{N}=1617$, skewness $=10.4$, S.E. $=.04$; respondents who did not consume alcohol in the past 30 days were excluded from analysis.

Source: ESPAD 2011

mean alcohol consumption is evident for Finnish boys, however in case of girls, there was a relatively large decline between the years 2003 and 2007. In addition, Table 3 reports the proportion of "strong drinkers", i.e. juveniles, who have consumed more than $100 \mathrm{cl}$ of $100 \%$ alcohol (the value of the alcohol index $>100$ ) in the past month. The trend with respect to strong drinkers is not entirely consistent with the above-mentioned trend of mean alcohol consumption. There is consistently around $7 \%$ of strong drinkers in the Finnish youth population. Whereas the number of strong drinkers gradually decreases among Finnish girls, there was an increase (2003-2007) followed by a decrease (20072011) in the proportion of strong drinkers in case of boys.

In the Czech Republic, there was a decline in mean alcohol consumption among teenagers who drink alcohol between 2003 and 2007, followed by an increase between 2007 and 2011 (see Table 3). This development corresponds also with the proportion of strong drinkers in the population of drinkers. The same can be said about the mean alcohol consumption among Czech boys and among girls. But while the proportion of strong drinkers among boys follows this trend as well, the proportion of strong drinkers among girls declines in time.

The relationship between mean alcohol consumption and the proportion of strong drinkers is, however, very strong (Pearson's $\mathrm{r}=.98, \mathrm{p}<.001$ ). Thus, we can conclude that a change in the mean consumption of alcohol is associated with a change in the proportion of strong consumers of alcohol in the population.

Next, we ran a series of linear regression models on log aggregate data. Linear regression with 12 data points (each point reflects one group of drinkers based on gender, country, and year) was used for each percentile group. The models explain a relatively large part of variance. The lowest value of $\mathrm{R}^{2}(.67)$ is found in the model that describes a group of drinkers at the $25^{\text {th }}$ percentile. The other $\mathrm{R}^{2}$ have values around .9 and explain approximately $90 \%$ of the variance in the data. 
Table 3: Mean alcohol consumption in the last 30 days and the proportion of strong drinkers between Czech and Finnish juveniles (2003, 2007, and 2011)

\begin{tabular}{|c|c|c|c|c|}
\hline Country/year & $n^{1}$ & \multicolumn{2}{|c|}{ Mean alcohol consumption ${ }^{2}$} & \multirow[t]{2}{*}{ Proportion of strong drinkers ${ }^{3}$} \\
\hline Finland & & & & \\
\hline 2003 & & Mean & S.E. & $\%$ \\
\hline Boys & 931 & 30.5 & 1.5 & 6.0 \\
\hline Girls & 789 & 42.7 & 3.2 & 8.6 \\
\hline Total & 1720 & 36.0 & 1.7 & 7.2 \\
\hline \multicolumn{5}{|l|}{2007} \\
\hline Boys & 1052 & 40.3 & 2.8 & 8.7 \\
\hline Girls & 1309 & 33.7 & 1.9 & 6.9 \\
\hline Total & 2361 & 36.6 & 1.6 & 7.7 \\
\hline \multicolumn{5}{|l|}{$\underline{2011}$} \\
\hline Boys & 831 & 45.9 & 3.9 & 7.0 \\
\hline Girls & 950 & 32.5 & 2.2 & 5.4 \\
\hline Total & 1781 & 38.6 & 2.1 & 7.2 \\
\hline \multicolumn{5}{|l|}{ Czech Republic } \\
\hline 2003 & & Mean & S.E. & $\%$ \\
\hline Boys & 1102 & 93.1 & 4.1 & 26.6 \\
\hline Girls & 1308 & 42.5 & 2.3 & 10.7 \\
\hline Total & 2410 & 65.6 & 2.3 & 18.0 \\
\hline \multicolumn{5}{|l|}{2007} \\
\hline Boys & 1356 & 78.8 & 3.9 & 21.5 \\
\hline Girls & 1551 & 45.6 & 2.2 & 12.2 \\
\hline Total & 2907 & 61.1 & 2.2 & 16.6 \\
\hline \multicolumn{5}{|l|}{$\underline{2011}$} \\
\hline Boys & 1539 & 86.8 & 3.3 & 26.2 \\
\hline Girls & 1528 & 42.3 & 2.0 & 12.0 \\
\hline Total & 3067 & 64.6 & 2.0 & 19.1 \\
\hline
\end{tabular}

Note: ${ }^{1}$ juveniles who consumed alcohol in the last 30 days; ${ }^{2}$ centiliters of $100 \%$ alcohol; ${ }^{3}$ juveniles who consumed more than $100 \mathrm{cl}$ of $100 \%$ alcohol in the last 30 days (alcohol index > 100).

Source: ESPAD

Table 4 presents regression coefficients of models for each group of drinkers and the results are also depicted in Figure 3. An increase in mean consumption in the population leads to an increase in alcohol consumption in all groups of consumers. The results are, thus, consistent with Skog's (1985) theory and support the proposition that alcohol consumption has a strong collective character, so that the entire population is consistently moving up/down the scale of consumption. However, the level by which the consumption increases is different among the groups. The lowest increase is found 
among light drinkers (P25). When mean consumption of the whole population doubles, the consumption of light drinkers increases 1.6 times. Conversely, the highest values of coefficients have groups of near heavy (P90) and heavy (P95) drinkers. When mean consumption of the population doubles, the consumption of these groups increases 2.4 times and 2.3 times respectively. Thus, unlike Skog's findings, we conclude that it is the group of near heavy drinkers and heavy drinkers who are more affected by a change in mean alcohol consumption in the whole population compared to the other drinking groups.

Table 4: Regression models for 5 groups defined by percentile values - group consumption (logged) by mean consumption (logged)

\begin{tabular}{|l|c|c|c|}
\hline percentile & B & beta & sig. \\
\hline P25 & .64 & .82 & .001 \\
\hline P50 & .87 & .93 & $<.001$ \\
\hline P75 & 1.10 & .98 & $<.001$ \\
\hline P90 & 1.27 & .98 & $<.001$ \\
\hline P95 & 1.19 & .97 & $<.001$ \\
\hline
\end{tabular}

Source: ESPAD

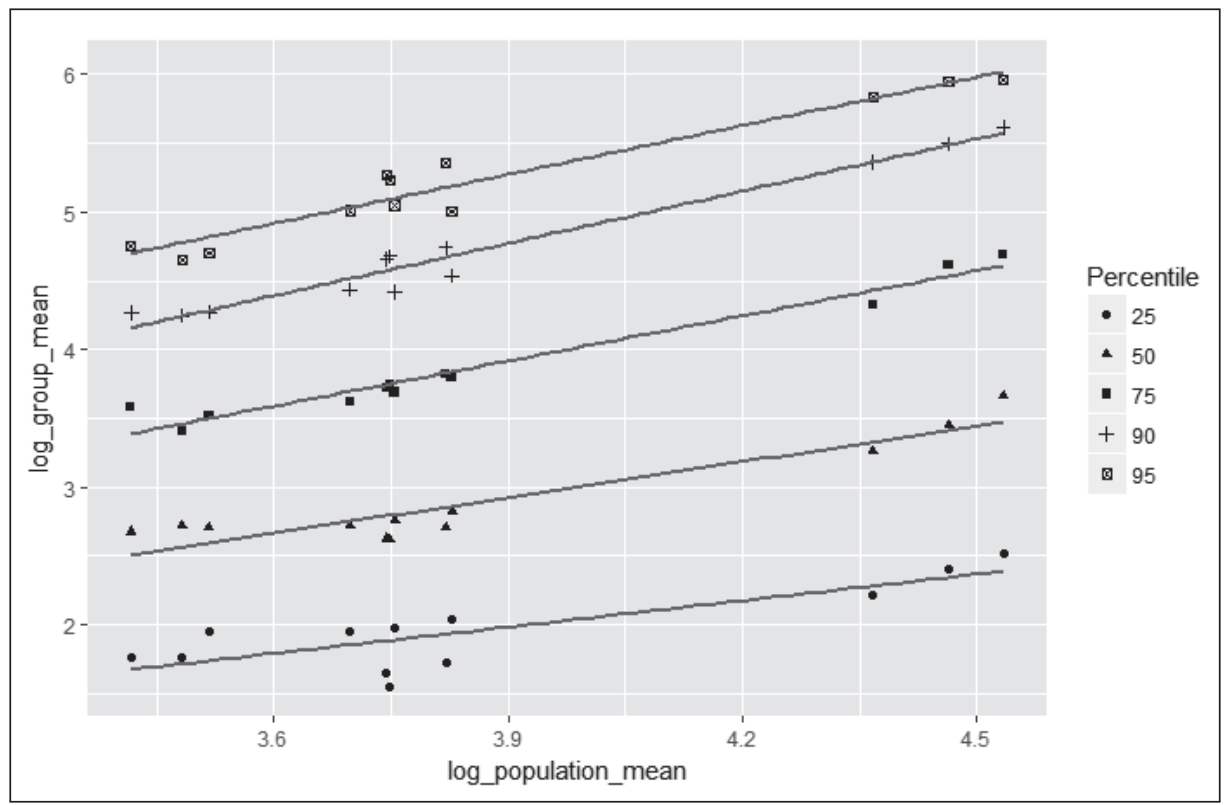

Figure 3: Mean consumption (axis $\mathrm{x}$ ) by percentile values (axis y) for 12 gender specific groups Note: Percentile values for the 25, 50, 75, 90, and 95 percentiles; log data reported Source: ESPAD

Finally, we conducted an analogous analysis on data from the ISRD study. This survey did not include information on the amount of alcohol consumed by juveniles, but only the number of occasions when they had drunk alcohol during the past month was re- 
ported. Data were aggregated into 8 groups by country, wave of ISRD and gender (shown in Table 5). The population mean of the frequency of alcohol consumption was calculated for each of the 8 groups. The procedure was identical as with the ESPAD data and included only juveniles who consumed alcohol in the past month.

The average numbers of drinking occasions for each group are reported in Table 5. There is an increase in frequency of alcohol consumption in both countries between 2006 and 2013, nevertheless it is steeper in the Czech Republic compared to Finland. Interestingly, gender differences are more pronounced in CZ, whereas they are negligible in Finland, with even somewhat higher mean value for girls than for boys in 2013.

Table 5: The average frequency of alcohol consumption in the last month by country and gender

\begin{tabular}{|c|c|c|c|c|}
\hline & & & $\mathbf{n}$ & Average number of drinking occasions \\
\hline \multirow[t]{6}{*}{ Czech Republic } & \multirow{3}{*}{ ISRD-2 (2006) } & Boys & 218 & 3.0 \\
\hline & & Girls & 193 & 2.3 \\
\hline & & Total & 411 & 2.7 \\
\hline & \multirow{3}{*}{ ISRD-3 (2013) } & Boys & 500 & 5.7 \\
\hline & & Girls & 546 & 4.7 \\
\hline & & Total & 1046 & 5.2 \\
\hline \multirow[t]{6}{*}{ Finland } & \multirow{3}{*}{ ISRD-2 (2006) } & Boys & 186 & 2.6 \\
\hline & & Girls & 223 & 2.4 \\
\hline & & Total & 409 & 2.5 \\
\hline & \multirow{3}{*}{ ISRD-3 (2013) } & Boys & 317 & 3.6 \\
\hline & & Girls & 395 & 3.9 \\
\hline & & Total & 712 & 3.8 \\
\hline
\end{tabular}

Source: ISRD

Results of linear reggression analysis are presented in Table 6 and depicted in Figure 4. The ISRD data show that an increase in mean frequency of alcohol consumption in population leads to an increase in the frequency of alcohol consumption at all levels of consumers. The smallest increase is visible in the group of light drinkers (P25). When mean frequency of consumption of a population doubles, the frequency of consumption of this group increases 1.9 times. Conversely, the highest values of coefficients have groups of near heavy (P90) and heavy (P95) drinkers. When mean frequency of consumption of the population doubles, the frequency of consumption of these groups increase 2.5 times and 2.7 times respectively. Thus, consistently with previous results from ESPAD, when there is an increase in the average frequency of alcohol consumption in a population, heavy drinkers have the largest increase in their frequency of alcohol consumption. 
Table 6: Regression models for 5 groups defined by percentile values - group consumption (logged) by mean frequency of consumption (logged)

\begin{tabular}{|l|c|c|c|}
\hline percentile & B & beta & sig. \\
\hline P25 & .89 & .94 & .001 \\
\hline P50 & 1.00 & .98 & $<.001$ \\
\hline P75 & 1.12 & .99 & $<.001$ \\
\hline P90 & 1.34 & .99 & $<.001$ \\
\hline P95 & 1.44 & .98 & $<.001$ \\
\hline
\end{tabular}

Source: ISRD

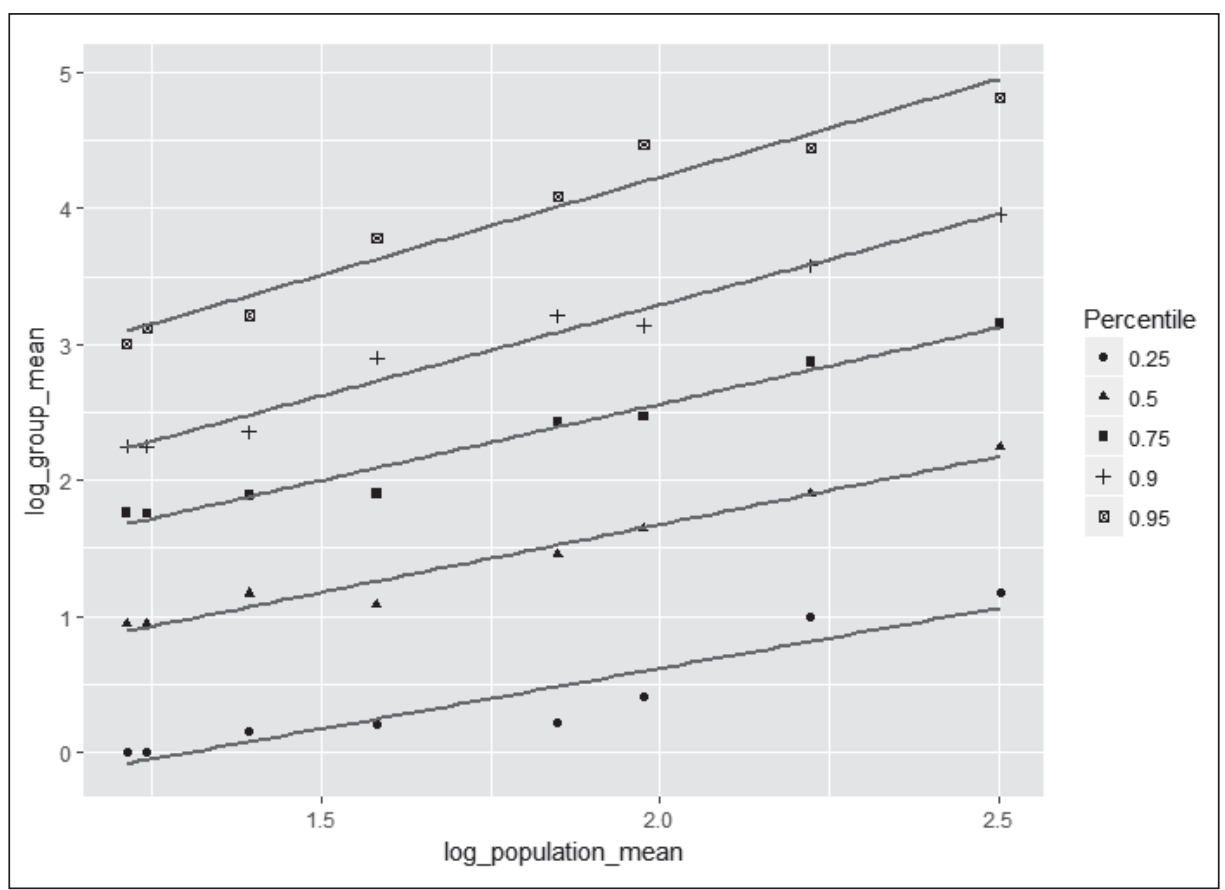

Figure 4: Mean frequency of consumption (axis $\mathrm{x}$ ) by percentile values (axis $\mathrm{y}$ ) for 8 gender specific groups

Note: Percentile values for the $25,50,75,90$, and 95 percentiles; log data reported

Source: ISRD

\section{Discussion}

The aim of this article was to describe the situation with respect to alcohol consumption among Czech and Finnish youth and to empirically test Skog's (1985) theory of the distribution of alcohol consumption. For this purpose, we used data from two large-scale international surveys for the Czech Republic and Finland. The results show that there 
are, indeed, considerable differences between Czech and Finnish juveniles in alcohol consumption and in its trend over the last years. Such variability is a convenient starting point for the test of Skog's theory which results are supportive of all three propositions. First, the distribution of alcohol consumption is strongly skewed to the right in both countries. Second, a strong relationship between mean alcohol consumption in the population and the proportion of strong drinkers has been found. Third, it has also been confirmed that a change in mean alcohol consumption in a population is associated with a change in alcohol consumption at all levels of consumers, from light to heavy drinkers.

However, unlike Skog's (1985) findings showing that an increase in mean alcohol consumption in a population has the largest impact on the increase in alcohol consumption among light drinkers (see also Brunborg, Bye, \& Rossow, 2014), in our analysis, it is the group of heavy drinkers (and near heavy drinkers) which is affected the most by such a change. Furthermore, our analysis of the ISRD data has revealed that Skog's (1985) theory is supported even if another indicator of alcohol consumption is used. In this case, the number of occasion in which alcohol was consumed in the last month, i.e. the frequency of alcohol consumption, yields comparable results as the analysis considering the amount alcohol consumed in the last month.

Presented analyses are, nevertheless, not without limitations. First, the exclusion of juveniles who did not consume alcohol in the last 30 days from analysis might be problematic as it comprises a high proportion of youth. We have decided for this approach as we have followed the lead by Brunborg, Bye, and Rossow (2014). However, it would be desirable to examine possibilities of including "non-consumers" in analysis as well. Second, another procedure overtaken from the study by Brunborg, Bye, and Rossow (2014) was a division of data into populations of girls and boys, likely because of an increase in the number of data points for linear regression models. Such division assumes that boys and girls are distinct groups which create separate networks of consumers and different standards for alcohol consumption. Although this assumption might be correct as adolescent girls and boys spend their leisure time rather separately, further analysis should confirm this assumption. Final limitation is the low number of data points in our linear regression models. For more accurate results, it would be useful to analyze data from more countries and/or using more time points.

Despite these limitations, we find the results as a contribution to the discussion of Skog's theory. Moreover, the results are directly comparable with the analysis of Norwegian data from ESPAD (Brunborg, Bye, \& Rossow, 2014). Findings presented in this article have clear implications with respect to prevention of youth alcohol abuse. As has been shown, if there is a change in mean alcohol consumption in a population, there is a change at all levels of consumption, but the largest change occurs among heavy drinkers. This conclusion is supportive of universal preventive efforts targeted at the whole youth population. Any success in reduction of the average alcohol consumption is also likely to substantially reduce the amount of alcohol consumed by heavy drinkers, thus reduce alcohol-related harm among youth as well. Furthermore, as Finland is obviously successful in reduction of youth alcohol consumption, their alcohol policy could be an inspiration for the Czech policy makers. Higher prices of alcohol, better age control before selling alcohol, strict punishments for selling alcohol to youth, reducing the number 
of advertisements in media and greater parental control are possible ways which could contribute to reduction of alcohol consumption of Czech youth.

\section{Acknowledgements}

1) This article includes data from a database produced within the European School Survey Project on Alcohol and Other Drugs (ESPAD). This article is written in the line with the rules for the use of the ESPAD database. The National Principal Investigator and Contact Person providing data for this study was Zuzana Podaná, Czech Republic, Charles University.

2) This article includes data from a database produced within the project International Self-Report Delinquency Study (ISRD). The dataset from ISRD-3 was kindly provided by the ISRD Steering Committee. The Contact Person providing data for this study was Zuzana Podaná, Czech Republic, Charles University.

\section{REFERENCES}

Bjarnason, T. (2006). Polarization in alcohol consumption among Icelandic adolescents, 1995-2003. Nordic Studies On Alcohol And Drugs, 23, 51-58.

Brunborg, G. S., Bye, E. K., \& Rossow, I. (2014). Collectivity of drinking behavior among adolescents: An analysis of the Norwegian ESPAD data 1995-2011. Nordic Studies On Alcohol, 31(4), 389-400.

Chomynová, P., Csémy, L., \& Mravčík, V. (2016). Evropská školní studie o alkoholu a jiných drogách (ESPAD) 2015. Zaostřeno, 2(5), 1-16. Retrieved from https://www.drogy-info.cz/data/obj _files/32196/734/zaostreno_2016-05_v03.pdf

Csémy, L., Lejčková, P., Sadílek, P., \& Sovinová, H. (2006). Evropská školní studie o alkoholu a jiných drogách 2003. Praha: Úřad vlády ČR. Retrieved from http://www.drogy-info.cz/data/obj _files/1665/361/espad_web.pdf

Čermáková, I. (2016). Konzumace alkoholu mládeží: testování Skogovy teorie distribuce alkoholové konzumace. Diplomová práce. Praha: Filozofická fakulta Univerzity Karlovy.

Gmel, G., \& Rehm, J. (2000). The empirical testability of Skog's theory of collective drinking behaviour. Drug and Alcohol Review, 19(4), 391-399.

Hibell, B., Guttormsson, U., Ahlström, S., Balakireva, O., Bjarnason, T., Kokkevi, A., \& Kraus, L. (2012). The 2011 ESPAD Report. Substance Use Among Students in 36 European Countries. Stockholm: The Swedish Council for Information on Alcohol and other Drugs. Retrieved from http://www.espad.org /uploads/espad_reports/2011/the_2011_espad_report_full_2012_10_29.pdf

Hallgren, M., Leifman, H., \& Andreasson, S. (2012). Drinking Less But Greater Harm: Could Polarized Drinking Habits Explain the Divergence Between Alcohol Consumption and Harms among Youth? Alcohol And Alcoholism, 47(5), 581-590.

ISRD3 Working Group (2013). Questionnaire ISRD3: Standard Student Questionnaire (ISRD3 Technical Report Series \#2). Boston, MA: Northeastern University, School of Criminology and Criminal Justice.

Junger-Tas, J., Marshall, I. H., Enzmann, D., Killias, M., Steketee, M., \& Gruszczynska, B. (2010). Juvenile delinquency in Europe and beyond. New York: Springer.

Kraus, L. et al. (2016). ESPAD Report 2015. Results from the European School Survey Project on Alcohol and Other Drugs. Luxembourg: Publications Office of the European Union. Retrieved from http:// www.espad.org/sites/espad.org/files/TD0116475ENN.pdf

Kubička, L., Csémy, L., Duplinský, J., \& Kožený, J. (1998). Czech men’s drinking in changing political climates 1983-93: a three wave longitudinal study. Addiction, 93(8), 1219-1230.

Livingston, M. (2008). Recent trends in risky alcohol consumption and related harm among young people in Victoria, Australia. Australian \& New Zealand Journal Of Public Health, 32(3), 266-271. 
Meier, P. (2010). Polarized drinking patterns and alcohol deregulation. Nordic Studies On Alcohol And Drugs, 27(5), 383-408.

Moravcová, E., Podaná, Z., \& Buriánek, J. (2015). Delikvence mládeže: trendy a souvislosti. Praha: Triton. Norström, T., \& Svensson, J. (2014). The declining trend in Swedish youth drinking: collectivity or polarization? Addiction, 109(9), 1437-1446.

Rossow, I., Mäkelä, P., \& Kerr, W. (2014). The collectivity of changes in alcohol consumption revisited. Addiction, 109(9), 1447-1455.

Skog, O. J. (1985). The Collectivity of Drinking Cultures: A Theory of the Distribution of Alcohol Consumption. British Journal Of Addiction, 80(1), 83-99.

World Health Organization. (2014). Global status report on alcohol and health 2014. Geneva, World Health Organization. Retrieved from http://apps.who.int/iris/bitstream/10665/112736/1/9789240692763 _eng.pdf?ua=1 